\title{
Co-option of male courtship signals from aggressive display in bowerbirds
}

\author{
Gerald Borgia* and Seth William Coleman \\ Department of Biology, University of Maryland, College Park, MD 20742-4415, USA
}

\begin{abstract}
The pre-existing trait hypothesis suggests that females evolve a mating preference for an already existing male trait. This hypothesis poses a simple resolution to Darwin's long-standing question of how elaborate, male display traits evolve. The frequently observed convergence of aggressive and courtship displays across a wide array of species provides the only current support for this hypothesis. Here we provide much more detailed supporting evidence from bowerbird skrraa calls used in aggression and courtship. Consistent with the pre-existing trait hypothesis we show that (i) putatively co-opted skrraa calls used in courtship and aggression are homologous, (ii) skrraa calls were used in aggression in bowerbirds before being used in courtship, (iii) historically, intense, aggressive-like courtship calls were present near the time of co-option, and (iv) bower types contemporaneous with co-option emphasize design features that provide females protection from the adverse effects of intense courtship displays. These results, plus evidence for a female preference for males with intense aggressive-like courtship skrraa calls, suggest that aggressive skrraa calls have been co-opted for use in male courtship display.
\end{abstract}

Keywords: co-option; pre-existing trait; bowerbirds; courtship signals; mate choice

\section{INTRODUCTION}

The evolution of extreme male displays was of considerable interest to Darwin and has been a significant issue in discussions of sexual selection over the past 20 years. A variety of models have been suggested that may explain these traits (see Andersson 1994). Models requiring the coevolution of male traits and female preferences (e.g. runaway and some versions of good genes) may not work because they require high genetic variation and genetic correlations that may be difficult to maintain in traits under selection (Turelli 1984; Borgia 1987; Nichols \& Butlin 1989, 1992; Breden et al. 1994). Pre-existing preference models that argue that male traits evolve in response to an already evolved but newly expressed female preference have been proposed as more realistic alternatives (e.g. Kirkpatrick \& Ryan 1991; Ryan 1998). The female preferences driving the evolution of male traits are thought to be side-effects of selection for other traits (Burley 1978) and are thus unlikely to produce good genes effects. Recent evidence from several studies showing a good genes effect have complicated this picture (Petrie 1994; Hasselquist et al. 1996; Wilkinson et al. 1998). One interpretation is that the requirements of coevolutionary models are not as onerous as has been suggested (e.g. Bakker \& Pomiankowski 1995). Another possibility that we consider here is that there are alternative hypotheses, like the pre-existing trait model, that are not coevolutionary, but are consistent with good genes selection.

The war propaganda (Borgia 1979) or more recently labelled pre-existing trait hypothesis (Berglund et al. 1996) proposes that females evolve preferences for already existing male display traits that are then co-opted for use in courtship. Most often, courtship displays are thought to be co-opted from aggressive displays. The hypothesis suggests that, in aggressive displays, physically superior males that have signals indicating their greater vigour

*Author for correspondence (borgia@umail.umd.edu). can scare off competitors and thereby avoid harm inflicted by even an inferior opponent in an escalated contest. These same signals may be used by females to evaluate a prospective mate's ability to provide genetic and/or proximate benefits reliably. Vigorous males that can scare off or intimidate competitors may have genes that will produce vigorous offspring. Females may also garner benefits such as protection from interference by other males (Borgia 1979) or a low probability of male transfer of diseases (Borgia \& Collis 1990). Because a preexisting male trait is already present, there is no requirement that it co-evolves with the female preference and, because the female preference evolves in response to advantages conferred by the trait, this type of mate choice brings benefits to choosing females.

Under the pre-existing trait hypothesis male traits are co-opted from use in one form of display for use in another. Co-option is widely recognized as important in contributing to the evolution of complex traits ranging from genetic architecture (Martin 1999) to helping kin (Hamilton 1966). The co-option of traits for male display has been discussed in the ethological literature (e.g. Schenkel 1956), but with emphasis on these traits as ritualized signals. Trivers (1972) refocused consideration of male display trait evolution on assessment of male quality, but, unlike earlier ethological work, co-option has received scant attention (e.g. Andersson 1994).

Currently, the only evidence for the pre-existence trait hypothesis comes from the observation of similarity between aggressive and courtship displays across a wide array of species (Beebe 1926; Loffredo \& Borgia 1986; Berglund et al. 1996; Mateos \& Carranza 1999). However, this similarity in courtship and aggressive calls could arise for other reasons, for instance if aggressive calls were co-opted from courtship calls.

In bowerbirds 'skrraa calls' are used in courtship and aggression and may provide an example of an aggressive display that was co-opted for use in courtship. In most bowerbird species courtship and copulation occur at a 


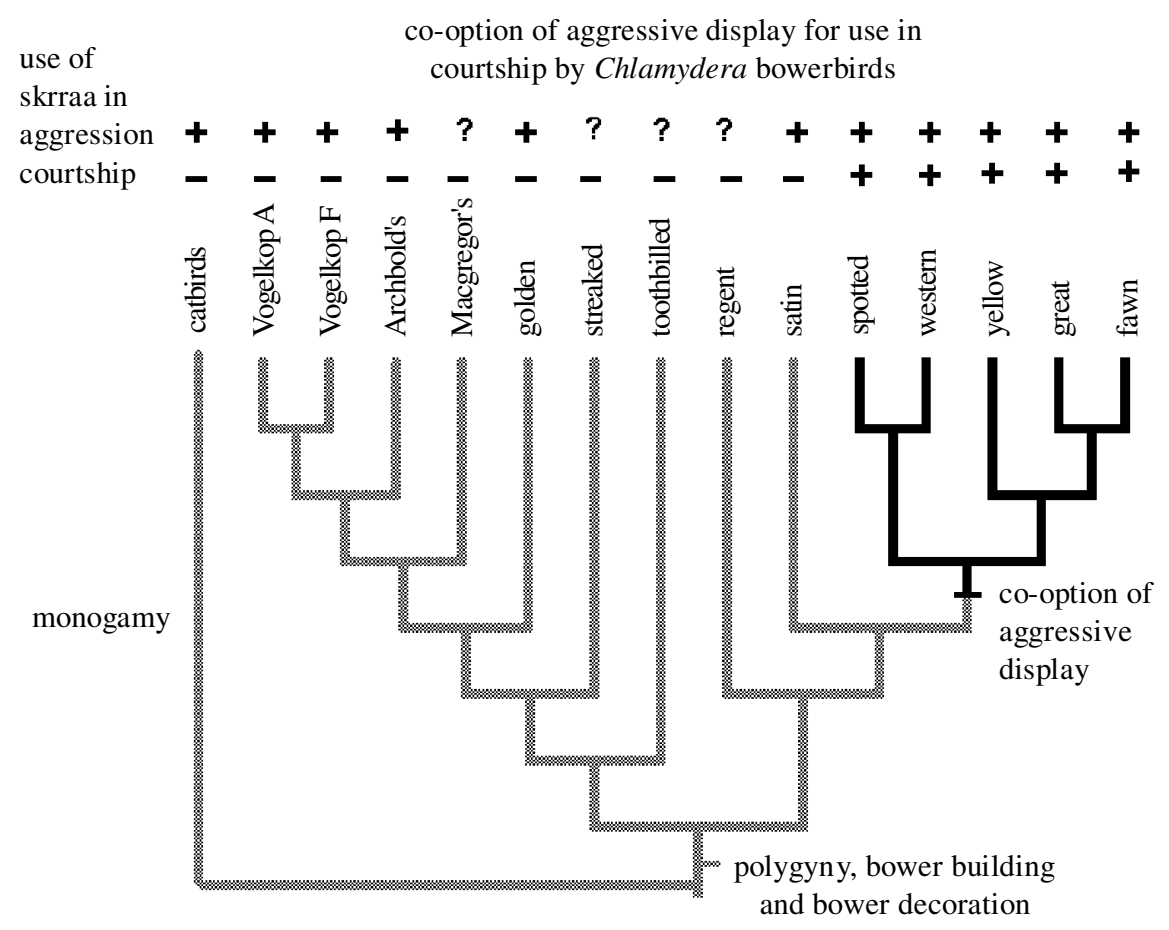

Figure 1. Phylogeny of the bowerbirds (Kusmierski et al. 1997) onto which the presence $(+)$, absence $(-)$ or presence unknown (?) of skrraa calls in aggressive and courtship displays are mapped. This mapping is conservative; the usual practice of deleting taxa with unknown states (e.g. Schluter et al. 1997) indicates that aggressive skrraa calls were present in the earliest bowerbirds and that skrraa calls were only later used in courtship coincident with the divergence of Chlamydera. If unknown states are classified as absences, satin bowerbird use of skrraa calls in aggression but not courtship still supports an earlier appearance of aggressive skrraa calls than courtship skrraa calls. Information on placement of western bowerbirds was provided by J. Bollback.

decorated display court adjacent to a stick structure called a bower. Males use skrraa calls in fights that result from encounters with other males at bowers (Borgia \& Gore 1986). Mate choice is focused on indicator traits that are part of the bower, display site (Borgia 1985) and vocal display (Borgia \& Presgraves 1998). Here we use phylogenetic and biogeographical information to test the hypothesis that courtship skrraa calls were co-opted from skraa calls used in aggression.

\section{METHODS}

Behaviours of 15 species and subspecies at bowers were monitored (Kusmierski et al. 1997) with a remote-controlled video camera system (Borgia 1995) that recorded courtship and, in some cases, aggressive behaviour at bowers. Microphones for sound recordings on video tape were placed at a constant distance $(1 \mathrm{~m})$ above the display court. Information on aggressive calls was obtained from videotaped aggressive encounters between males and from direct observations, but this information was not available for species that had only infrequent malemale aggressive interactions near their bowers. More intense calls are predicted to have a longer call duration and higher peak frequency (Morton 1977; Ryan et al. 1990). Call durations, peak frequencies and relative amplitudes were measured using a Kay model DSP5500 sonograph (Kay Elemetrics Corporation, Pine Brook, NJ, USA). Skrraa call amplitude was used for mapping call intensity because it provides a general measure that can be used across call types (co-opted and otherwise) and, thus, allowed inclusion of outgroup species in trait maps. The relative amplitude of courtship calls could be reliably measured because males called from a fixed distance from the microphone and because of the wide variation in amplitude between species. The high correlation of courtship call amplitude with peak frequency $\left(r_{\mathrm{p}}=0.99\right.$ and $\left.p=0.01\right)$ and call duration $\left(r_{\mathrm{p}}=0.94\right.$ and $p=0.017$ ) within the Chlamydera bowerbirds supports the suggestion that amplitude differences between species are consistently measurable and meaningful. Thus, courtship call amplitude is used as a measure of call intensity unless otherwise indicated. Amplitude comparisons were not made for aggressive calls because these were not measured at constant distances from the microphone. Calls from five males from each species were used in comparisons whenever possible. The statistical analyses used regression, Monte Carlo simulation and the Proc MIXED module of SAS (SAS Institute, Inc. 1999) allowing analysis of unbalanced, nested comparisons. Maximumparsimony (MP) and maximum-likelihood (ML) ancestor reconstructions were developed with the MacClade (Maddison \& Maddison 1992) and ANCML (Schluter et al. 1997) programs, respectively, using genetic distances provided by Kusmierski et al. (1997). ML reconstructions used two state variables and statistical significance was shown by absence of overlap of the confidence interval (95\% or greater) with the midpoint value. The PDAPT (Garland et al. 1993) program was used for independent contrasts of variables in comparisons among Chlamydera. The small sample size resulted in an undefined $F$-statistic. However, plots of transformed variables showed slopes near unity with low variation. This result, the strong association of bower and call characters with habitat conditions and general lability of bowerbird display traits (Kusmierski et al. 1997) suggest that phylogenetic effects on these characters were not large. The putative position of co-option of courtship display was determined by reconstruction of ancestor traits using MP. 
(a)
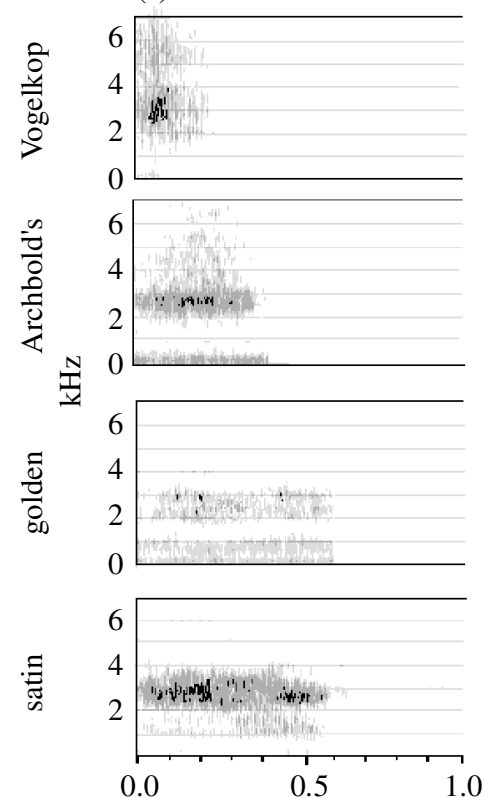

(b)
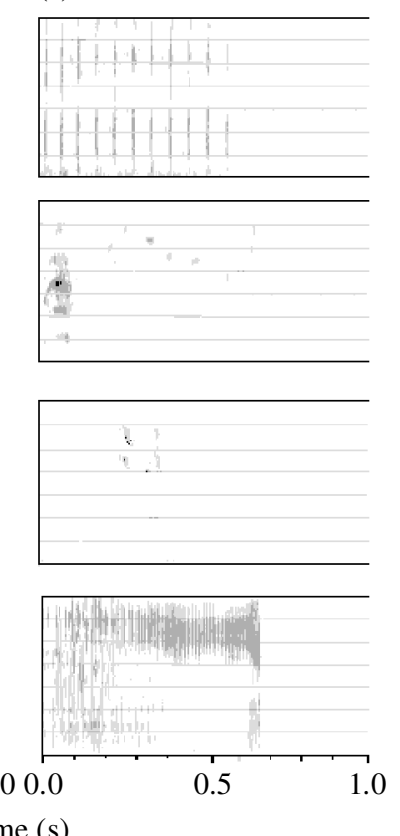

(c)
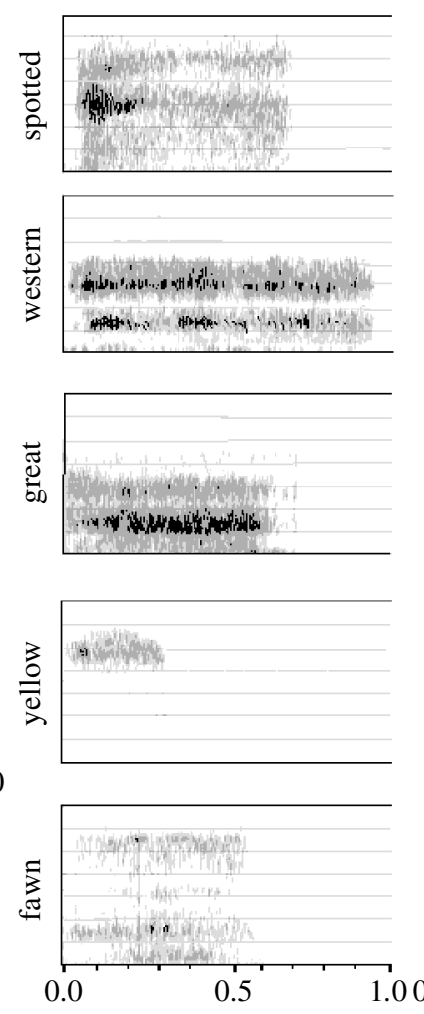

$(d)$
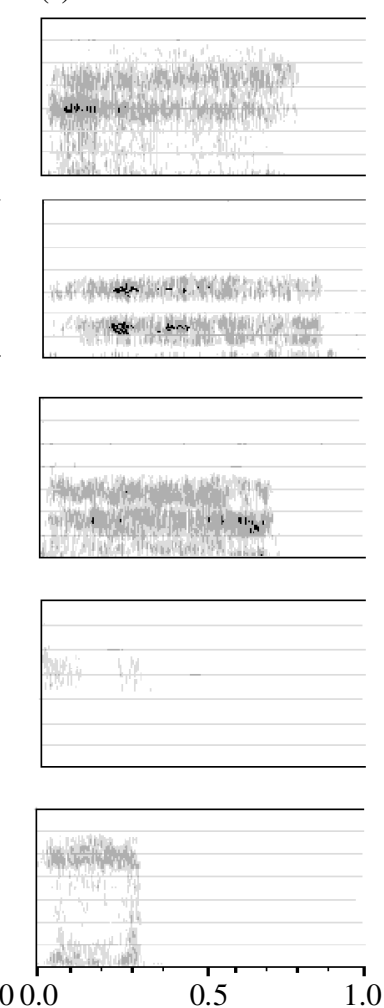

time (s)

Figure 2. Audiospectrograms of $(a)$ aggressive and $(b)$ courtship calls of pre-co-option species and $(c)$ aggressive and $(d)$ courtship calls of post-co-option species. Broadband noisy calls are used as aggressive calls across species and are used in courtship only in post-co-option species. Overtones contribute to some between-species variation of these calls, but do not obscure their fundamental similarity. The use of broadband aggressive calls as courtship calls only in post-co-option species but not in pre-co-option species is unlikely by chance (Monte Carlo simulation, $p<0.0016$ ).

\section{RESULTS AND DISCUSSION}

Phylogenetic tests have been used to evaluate sexual selection hypotheses (e.g. Basolo 1995; Johnson 1999) and can also be used to test for differences in the timing of the appearance of skrraa call functions in aggression and courtship. We mapped skrraa call use onto a robust phylogeny derived from cytochrome $b$ sequence information (Kusmierski et al. 1997). If aggressive calls evolved first, species using skrraa calls in aggression and courtship should be nested within a larger group using them only in aggression. This mapping shows that skrraa calls are used in courtship and aggression by Chlamydera bowerbirds only, with their nearest sister group (satin bowerbirds) and other bowerbirds using skrraa calls in aggression only (figure 1). This nested occurrence of courtship skrraa calls within the group of species using skrraa calls in aggression suggests that the use of skrraa calls in aggression preceded their use in courtship. This result indicates that the alternative possibility of aggressive calls being co-opted from courtship calls is incorrect.

The pre-existing trait hypothesis suggests that courtship displays in putatively post-co-option species should be homologues of aggressive calls. All species in our sample of bowerbirds used broadband aggressive calls (figure 2). Courtship calls of putatively pre-co-option species are highly variable with predominantly FM tonal elements, but post-co-option (Chlamydera) species use broadband skrraa calls that are very similar to aggressive calls. Peak frequency and duration are consistently measurable aspects of calls that have been predicted to be lower and shorter in aggressive-like calls, respectively (Morton 1977; Ryan et al. 1990). Aggressive calls of preco-option species and the courtship and aggressive calls of post-co-option Australian Chlamydera are similar in having a lower peak frequency and longer duration than courtship calls of pre-co-option species (figure 3).

The pre-existing trait hypothesis claims that co-option occurs because females can assess males giving aggressivelike calls more effectively and predicts that initially co-opted calls were intense. Display call intensity varies among the post-co-option Chlamydera species with the spotted, great and western bowerbirds from Australia having higher amplitudes $(t=27.3$, d.f. $=23$ and $p<0.0001)$, lower peak frequencies $(t=-7.16$, d.f. $=23$ and $p<0.001)$ and longer call durations $(t=6.53$, d.f. $=23$ and $p<0.001)$ than fawn and yellow-breasted bowerbirds from New Guinea. Phylogenetic reconstruction of display intensity allows a test of whether courtship skraa calls were of low or high intensity at the time of co-option. MP (Maddison \& Maddison 1992) analysis of call intensity using information on courtship call amplitude from extant species (figure 4 insert) is consistent with the hypothesis that intense skrraa calls are ancestral, but this inference is not amenable to statistical test. ML ancestor reconstruction (Schluter et al. 1997) using the same information produced a similar result showing a $74 \pm 38 \%$ probability that ancestral calls are intense, but the large error commonly 

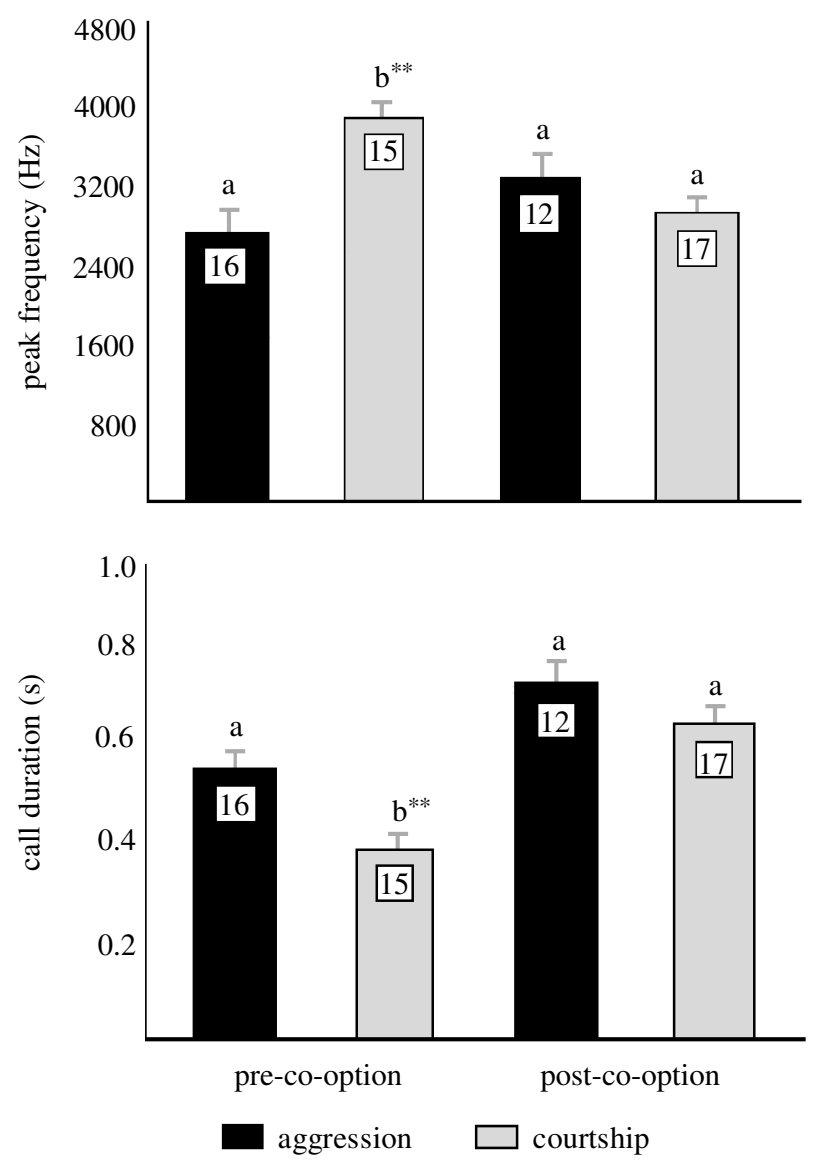

Figure 3. Comparisons of pre-co-option (four species shown in figure 2) and Australian Chlamydera bowerbirds with highintensity displays (spotted, great and western) for peak frequency and call duration. Bars represent means \pm s.e. and $n$ is given on the bars. Bars with different letters are significantly different $(<0.05)$ and double asterisks indicate differences greater than 0.01 . As predicted by the pre-existing trait hypothesis, pre- and post-co-option aggressive calls and coopted courtship calls did not differ, but pre-co-option courtship calls were different for both peak frequency and call duration. Only Australian Chlamydera are used here because these species are most representative of the co-opted call type (see $\S 3)$.

associated with ML reconstructions makes obtaining significance difficult (Schluter et al. 1997). Defining character states of nodes on the tree closer to the ancestor can reduce this large error. We used independent information on species' ranges in order to characterize nodes closer to the occurrence of co-option. The outgroup species, satin bowerbirds, is geographically adjacent to spotted and great bowerbirds, suggesting that the initial divergence from satin bowerbirds led to one of these other Australian species (figure 4). New Guinea species have uniquely raised bowers built on massive stick piles that level the display court on the steep slopes where they commonly build bowers (Borgia \& Presgraves 1998). Chlamydera species with stick piles have smaller courts (Monte Carlo simulation, $p<0.03$ ) and species with small courts have less intense displays $\left(r^{2}=0.97, F_{1,3}=105\right.$ and $\left.p<0.002\right)$. Males on small courts are constrained from modulating the negative effects of intense displays by moving away from the female (Borgia \& Presgraves 1998) resulting in a

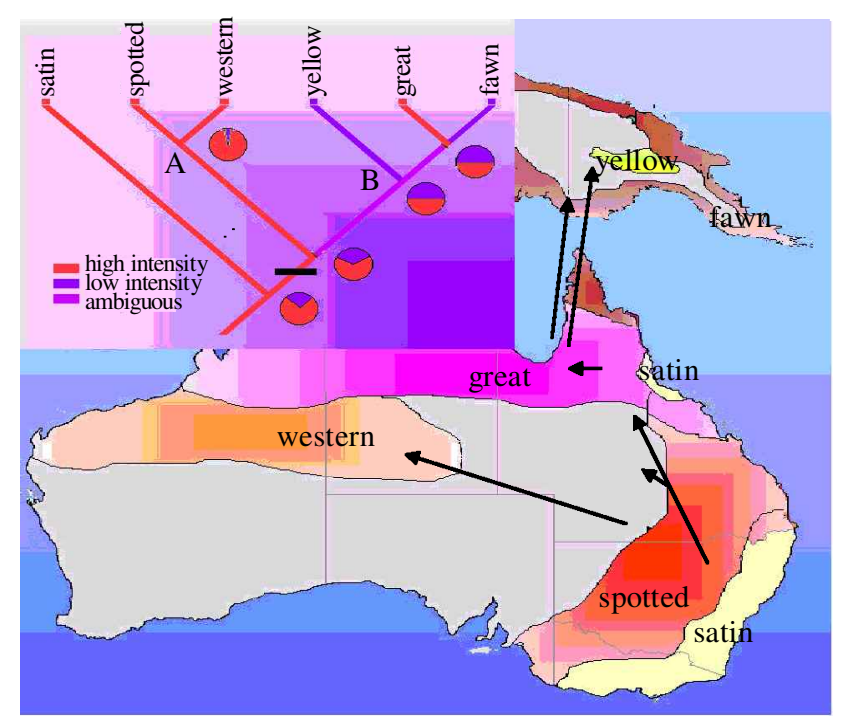

Figure 4. Map of species' ranges of Chlamydera and satin bowerbirds. The nearness of satin bowerbirds to spotted and great bowerbirds suggests that initial divergence was to one of these two species. Insert: maximum-parsimony plot

(Maddison \& Maddison 1992) of display intensity as measured by call amplitude using tip information (see the text). A black bar indicates the occurrence of co-option. Pie diagrams indicate maximum-likelihood probabilities (Schluter et al. 1997) in ancestor state reconstructions using tip information. A and B identify nodes that a second and more complete maximum-likelihood analysis using biogeographical and bower comparisons suggested should be characterized as having a high-intensity display.

low mean display intensity. The flat terrain that favours large display courts and intense displays of Australian Chlamydera has not changed since they diverged from satin bowerbirds (Galloway \& Kemp 1981). Thus, an ML reconstruction in which nodes close to the co-option event (nodes A and B in figure 4 insert) are characterized as having a high-intensity display indicates that high amplitude courtship calls were present at the time of cooption $(p<0.001)$. This conclusion is also supported by an analysis using an MP mapping of the peak frequency of aggressive skrraa calls. Aggressive calls of the New Guinea Chlamydera are unique in having high peak frequencies. Combined with evidence of the strong similarity of courtship and aggressive calls in post-co-option species, these results indicate that co-opted courtship calls were of high intensity and the low intensity courtship calls were derived in New Guinea.

Bowers in species with an intense co-opted display show unique adaptations in addition to differences in court size which mitigate the repelling effects of their displays (Borgia 1995). The appearance of these bower modifications may explain how originally aggressive displays that evolved to repel could have become attractive to females. Male spotted bowerbirds court the female through a unique see-through wall that forms a protective screen rather than at the bower entrance as in other species with similar bower types. Females prefer males with an intense display at intact spotted bowerbird bowers, but experimental removal of bower walls causes 
males to reduce their display intensity suggesting that an intense display is effective only when females are protected by the bower wall (Borgia \& Presgraves 1998). Long bowers of great bowerbirds separate males from females during courtship and may also provide threat reduction. Australian Chlamydera are unique among bowerbirds in their use of open habitats rather than forest and forest edges (Chaffer 1984). The shift to more open habitat may have provided space for expansive bowers and courts that enabled males to modulate intense displays and allowed the co-option of aggressive displays for use in courtship.

The pre-existing preference hypothesis (Burley 1978; Ryan et al. 1990) is an important alternative hypothesis not requiring trait and preference coevolution, but it is an unlikely explanation for skrraa call evolution. Skrraa call use in aggression is widespread among bowerbirds. Male bowerbirds are exceptional vocal mimics that incorporate other species' calls into their courtship vocalizations (e.g. Loffredo \& Borgia 1986; G. Borgia, personal observation). Thus, if their own aggressive skrraa calls were attractive to females in pre-co-option species as predicted by the pre-existing preference hypothesis, then males of these species should have incorporated them into their courtship display. Their absence suggests that females in pre-co-option bowerbirds do not find skrraa calls attractive. The pre-existing preference hypothesis also assumes that female preferences evolve as non-adaptive side-effects (Burley 1978), for example, by pleiotropy and that these preferences parallel those of the selected trait. For instance, a foraging preference for blue berries that incidentally alters female mating preferences for the colour of their mate's plumage should cause females to favour blue not red males as mates. In contrast, skrraa calls used in aggressive displays are repelling, but these same calls are attractive in Chlamydera courtship. The pre-existing trait hypothesis posits an independent, adaptive cause for female preferences driving the co-option of the male trait that can explain this reversal from repulsion to attractiveness. This is not explained by the pre-existing preference hypothesis.

Special thanks for advice and comments are due to B. BrittanPowell, L. Chao, R. Crozier, R. Dooling, T. Garland, B. Lohr, C. Mitter, G. Patricelli, K. Shaw, J. Lauridsen, A. Uy, G. Wilkinson and T. Wright. A. Uy, B. Rossi and M. Egeth provided technical assistance. Financial support was provided to G.B. by the National Science Foundation (USA), National Geographic Society, the Christensen Research Institute and the University of Maryland. Permits and access to parks were provided by the Australian Bird and Bat Banding Scheme, Lembage Ilmu Penegetahuan Indonesia, Indonesia, Papua New Guinea Forest and Conservation and the governments of Queensland, New South Wales and Western Australia, Australia. Thanks are due to the many field assistants who contributed to data collection and property owners who allowed access to their land.

\section{REFERENCES}

Andersson, M. 1994 Sexual selection. Princeton University Press.

Bakker, T. C. M. \& Pomiankowski, A. 1995 The genetic basis of female mate preferences. F. Evol. Biol. 8, 129-171.

Basolo, A. 1995 Phylogenetic evidence for the role of a preexisting bias in sexual selection. Proc. R. Soc. Lond. B 259, 307311.
Beebe, W. 1926 Pheasants, their lives and homes, vol. 2. Garden City, NY: Doubleday, Page \& Co.

Berglund, A., Bisazza, A. \& Pilastro, A. 1996 Armaments and ornaments: an evolutionary explanation of traits of dual utility. Biol. 7. Linn. Soc. 58, 385-399.

Borgia, G. 1979 Sexual selection and the evolution of mating systems. In Sexual selection and reproductive competition (ed. M. Blum \& A. Blum), pp. 51-58. New York: Academic Press.

Borgia, G. 1985 Bowers as markers of male quality: test of a hypothesis. Anim. Behav. 35, 266-271.

Borgia, G. 1987 A critical review of sexual selection models. In Sexual selection: testing the alternatives (ed. J. Bradbury \& M. Andersson), pp. 55-66. New York: Wiley.

Borgia, G. 1995 Threat reduction as a cause of differences in bower architecture, bower decoration, and male display in two closely related species of bowerbirds: Chlamydera nuchalis and C. maculata. Ети 95, 1-12.

Borgia, G. \& Collis, K. 1990 Parasites and bright male plumage in the satin bowerbird (Ptilonorhynchus violaceus). Am. Zool. 30, 279-285.

Borgia, G. \& Gore, M. 1986 Sexual competition by feather stealing in the satin bowerbird (Ptilonorhynchus violaceus). Anim. Behav. 34, 727-738.

Borgia, G. \& Presgraves, D. C. 1998 Coevolution of elaborated male display traits in the spotted bowerbird: an experimental test of the threat reduction hypothesis. Anim. Behav. 56, 1121-1128.

Breden, F., Geherardt, C. \& Butlin, R. K. 1994 Female choice and genetic correlations. Trends Ecol. Evol. Biol. 9, 343.

Burley, N. 1978 Sexual selection for aesthetic traits in species with parental care. Am. Nat. 127, 415-445.

Chaffer, N. 1984 In quest of bowerbirds. New York: Rigby.

Galloway, R. \& Kemp, E. 1981 Late Cainozoic environments in Australia. In Ecological biogeography of Australia (ed. A. Keast), pp. 52-77. Boston, MA: W. Junk.

Garland, T., Dickeman, A., Jones, C. \& Jones, J. 1993 Phylogenetic analysis of covariance by computer simulation. Syst. Biol. 42, 265-292.

Hamilton, W. D. 1966 The genetical evolution of social behavior. 7. Theor. Biol. 7, 1-52.

Hasselquist, D., Bensch, S. \& Von Schantz, T. 1996 Correlation between male song repertoire, extra-pair paternity, and offspring survival in the great reed warbler. Nature 381, 229232.

Johnson, K. P. 1999 The evolution of bill coloration and plumage dimorphism supports the transference hypothesis in dabbling ducks. Behav. Ecol. 10, 63-67.

Kirkpatrick, M. \& Ryan, M. 1991 The evolution of mating preferences and the paradox of the lek. Nature 350, 33-38.

Kusmierski, R., Borgia, G., Uy, A. \& Crozier, R. H. 1997 Labile evolution of display traits in bowerbirds indicates reduced effects of phylogenetic constraint. Proc. R. Soc. Lond. B 264, 307-313.

Loffredo, C. A. \& Borgia, G. 1986 Sexual selection, mating systems, and the evolution of avian acoustical displays. Am. Nat. 128, 773-794.

Maddison, W. \& Maddison, D. 1992 MacClade 3.04. Framingham, MA: Sinauer.

Martin, A. 1999 Increasing genomic complexity by gene duplication and the origin of vertebrates. Am. Nat. 154, 111-128.

Mateos, C. \& Carranza, M. 1999 The effects of male dominance and courtship display on female choice in the ringnecked pheasant. Behav. Ecol. Sociobiol. 45, 235-244.

Morton, E. S. 1977 On the occurrence and significance of motivational-structural rules in some bird and mammal sounds. Am. Nat. 111, 855-869. 
Nichols, R. A. \& Butlin, R. K. 1989 Does runaway sexual selection work in finite populations? F. Evol. Biol. 2, 299-313.

Nichols, R. A. \& Butlin, R. K. 1992 Sexual preference and genetic correlations. Trends Ecol. Evol. 7, 29-30.

Petrie, M. 1994 Improved growth and survival of offspring of peacocks with more elaborate trains. Nature 371, 598-599.

Ryan, M. 1998 Sexual selection, receiver biases, and the evolution of sex differences. Science 281, 1999-2003.

Ryan, M., Fox, J., Wilczynski, W. \& Rand, A. 1990 Sexual selection for sensory exploitation in the frog Physalaemus pustulosus. Nature 343, 66-67.

SAS Institute, Inc. 1999 SAS users' guide, v. 8 . Cary, NC: SAS Institute, Inc.
Schenkel, R. 1956 Zur Deutung Balzleistungen einiger Phasianiden und Tetraoniden. Ornithol. Beobachter 53, 182-201.

Schluter, D., Price,T., Mooers, A. O. \& Ludwig, D. 1997 Likelihood of ancestor states in adaptive radiation. Evolution 51, 1699-1711.

Trivers, R. 1972 Parental investment and sexual selection. In Sexual selection and the descent of man 1871-1971 (ed. B. Campbell), pp. 136-179. Chicago, IL: Aldine.

Turelli, M. 1984 Heritable genetic variation via mutationselection balance: Lerch's zeta meets the abdominal bristle. Theor. Pop. Biol. 25, 138-193.

Wilkinson, G. S., Presgraves, D. C. \& Crymes, L. 1998 Male eye span in stalk-eyed flies indicates genetic quality by meiotic drive suppression. Nature 391, 276-279. 